\title{
Intellectual Paradigm of Artificial Vision: From Video-Intelligence to Strong Artificial Intelligence
}

\author{
E. M. Yarichin \\ Laboratory of brain-like technologies \\ "NeocorTek Lab" (LLC) \\ Krasnoyarsk, \\ Russian Federation
}

\author{
V. M. Gruznov \\ Trofimuk Institute of Petroleum Ge- \\ ology and Geophysics \\ Siberian Branch of Russian \\ Academy of Sciences \\ Novosibirsk, Russian Federation
}

\author{
G. F. Yarichina \\ Institute for Business Processes and \\ Economics Management \\ Siberian Federal University, SFU \\ Krasnoyarsk, Russian Federation
}

\begin{abstract}
A new (post-Shannon) informational approach is suggested in this paper, which allows to make deep analysis of nature of the information. It was found that information could be presented as an aggregate of quantitative (physical) and qualitative (structural) components to be considered together. It turned out that such full information theory could be efficiently used as the guiding theory at modeling of video-information recognition, perception and understanding. These hierarchical processes are solving the intellectual tasks step-by-step for formation of the corresponding video-information evaluation and also represent a strong interactions-measurements videoinformation's ensuring adequacy of these assessments. That is why there is a need to build corresponding video information macro-objects (video-thesauruses) on every level of hierarchy of artificial vision system, which are formed by training (selftraining) and form together an upward hierarchy of qualitative measuring scales. The top of this hierarchy is video-intelligence. Information theory of artificial intelligence is a logical development of new information approach from analysis to synthesis. Further "analysis through synthesis" allows establishing the informational nature and structure of not only video-intelligence, but also strong artificial intelligence, which for video-intelligence constitute as intellectual suprasystem.
\end{abstract}

Keywords-Gauge approach; fibration space; informational hypergraph; video-intelligence; strong artificial intelligence

\section{INTRODUCTION}

Intuitive representation of intelligence as some informational macro-object that a perform information processing for the purpose of her understanding led to deep revision of classical (by Shannon) informational approach. As this approach was quite heuristic one, the goal was to build an overall view of surrounding material world properties using the actual advances in fundamental physics and mathematics without intuitive and heuristic aspects. Actually, the quantitative classical approach to information did not meet the needs of the researchers who deals with the tasks which qualitative nature became more obvious. Such tasks as recognition, perception and understanding can be example. If of usage of the quantitative approach to information at recognition tasks is somehow applicable, then in more complicated informational tasks of perception and understanding it is totally unhelpful. Any attempts to improve the classical informational approach by concept of information's value and other intuitive and heuristic aspects did not lead to anything.

That is why, post Shannon approach the information was suggested by one of the authors (E.M. Yarichin) of current article. This practical physics-mathematics approach let to clearly realize all the depth of information paradigm, to obtain the correct definition and to find out the structure of information. Information is turned out to be presented as a system of physical (quantitative) and structural (qualitative) components strongly interacted with each other that are always to be considered together. Quanta of physical and structural informational components are fundamentally differing from each other. Quanta of physical component are unstructured (zerodimensional, similar to the point). In its turn, structural component quanta or, otherwise, local qualts could be considered as infinitesimal univariate quantities determined in onedimensional space and due to this fact have a certain orientation. In small dimension world's spaces local qualts could be considered as the most natural elements to synthesize onedimensional global 1-qualts, then two-dimensional global 2qualts based on the previous ones, as well as global 3-qualts and 4-ones further.

Thus, as for full information (both physical and structural), known as video-information, its analysis for artificial vision is turned out to be a very important and obligatory stage, but not a sufficient one. Actually, if analysis is presented as investigation method of dividing unit on its parts, than synthesis as investigation method to merge divided parts and terms which were obtained during the analysis is more important when forming video informational evaluations. In other words, if post Shannon approach to information is an analysis, it takes to establish a dual approach to information, i.e. a synthesis to provide a forming upward hierarchy for of evaluations videoinformation in artificial vision.

\section{The GAUGE APPROACH TO VIDEO-INFORMATION PROCESSES IN THE FIBRATION FORMALISM}

Video-information is inherent in any material system formed by a strong interaction (interaction-measurement) of its own (unary) or mutual (binary) components [1]-[4]. These components of video-information must be comparable physically and structurally and the video-information itself could be interpreted in two ways. On the one hand, video-information is 
the only keep geometrophysical quantity (in the form of a second-order tensor) in the corresponding material system and, as a result, is taken as a source of its video-informational (geometrophysical) field (active, passive or activated). On the other hand, video-information is considered as geometrophysicsal measure of interaction-measurement of the physical and structural components of the corresponding material system. This dynamic aspect of video-information is a consequence of "gauge principle" based on the idea of gauge invariance.

In practice, the method of video informational field is carried out by the weak field approximation only, what is explain- ing by natural reduction of video-informational field during its paraxial space-time propagation from the source (active and/or activated video-information) to video sensor. Moreover, the weak video informational field could be considered as a gauge field [2], [3]. Due to weak informational field's ambiguity (gauge arbitrariness) the calibration of the video-receiver of the vision system is necessary. Only then will this geometrophysics measuring instrument uniquely form an estimate of weak video information in the form of an image (Fig. 1).

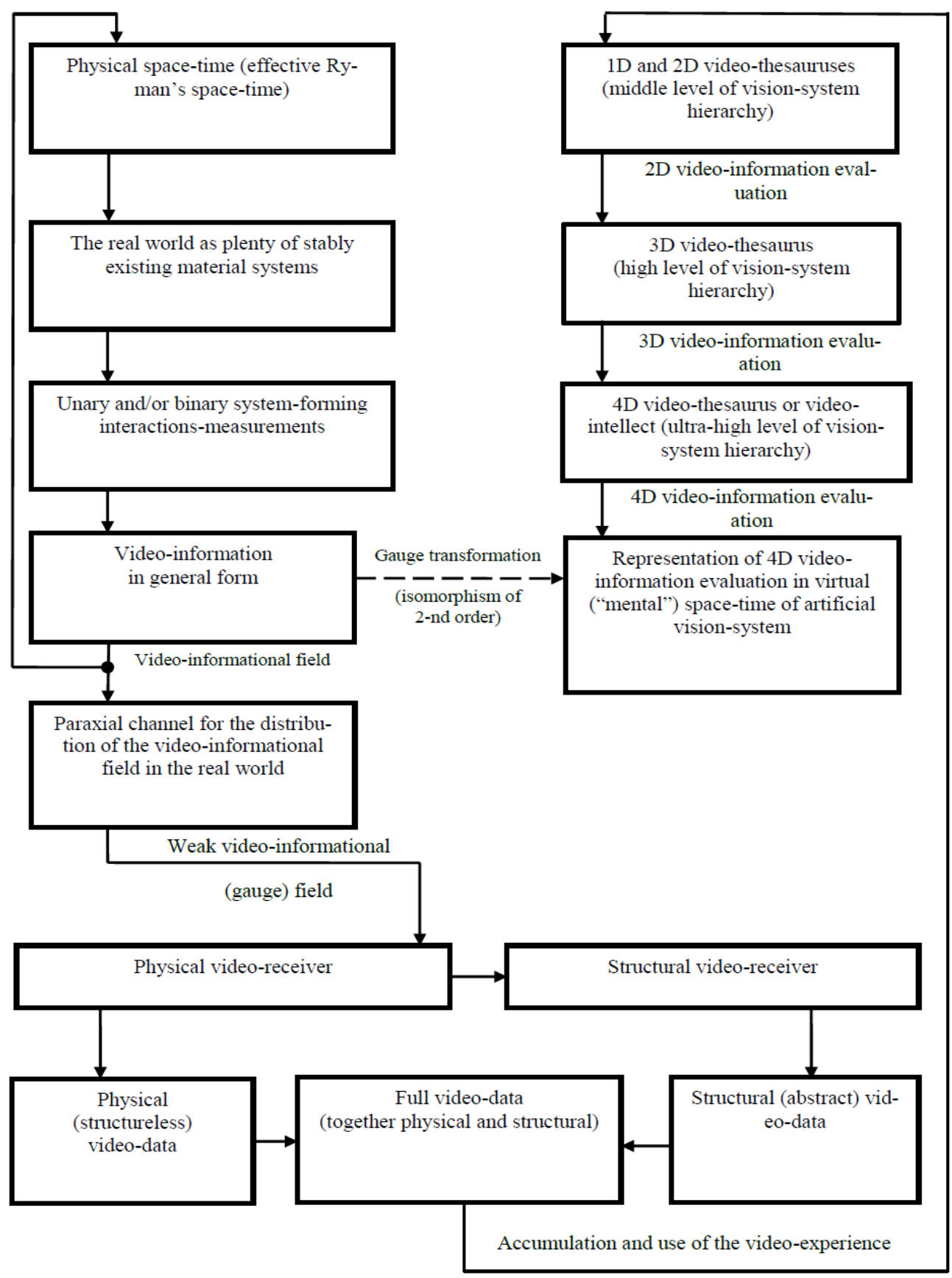

Fig. 1. General structural and functional scheme of artificial vision processes: the formation of video-information, the weakening of the video-information field, the restoration of video-information and the representation of its evaluation in the "mental" space of the artificial vision system. 
Weak informational field taken by video receiver is determined from its aperture's dimensions and initiates the process of artificial vision (Fig. 1). Besides, physical and structural components of video-receiver provide the analysis of physical and structural components of weak video-information respectively which is collated to this weak field so it has a weak (coarse) topology. Therefore, the weak video informational evaluation obtained is presented as obvious two-dimensional image (in the form of a 2-manifold) and also is the base to form a more advanced representation about this video-information at virtual ("mental") space with large number of dimensions. Corresponding abstract (e.g. 3D) image has a stronger (thin) topology, which could be presented by bundle on two-dimensional items, each one of them is an image of a studied object in a certain orientation concerning video-receiver.

The process of Images formation could be considered as weak video-information evaluation obtained by weak field analysis as a gauge field. From this point of view, images forming could be considered as gauge transformation according to gauge theory. As is known, such transformation does not affect on physical object's characteristics that could be studied and measured so it provides the adequacy of weak videoinformation and of evaluation the weak video-information.

As gauge transformations are supra-coordinated transformations they don't act on vectors and tensors of space-time but act on the spaces of internal characteristics of videoinformational fields [5]-[7]. Therefore, the geometry determined by the gauge fields is not space-time geometry, but a more General kind - a fibration space.

Thus, gauge approach to video-information could be formed due to local invariance principle corresponded to compensation circuit of gauge theory as well as within the framework of common description for video informational processes in terms of fibration. The fibration is presented as powerful tool for going out of traditional gauge theory. This could be the method formalization modeling of systems with the help of fibrations what is especially important for those systems where usual methods cannot be applied [7]-[9].

\section{VIDEO-INFORMATIONAL APPROACH TO ARTIFICIAL VISION}

The theory of information in its modern form is dedicated to the problems of information processing and transmission with redundancy, noise, losses and coding taken into account. Classical theory of information hasn't consider the fundamental problem of information creation and distinguish not take into consideration what could be referred to information and what to senseless and noise [10]-[14]. In addition, the information's forming it is assumed the prerogative of the third party system or suprasystem. This approach, allowable at communication theory, makes it hard to analyze and research processing systems of full information when not only quantitative (physicals) but also qualitative (structural: geometrical, logical) information's characteristics determine the end processing result and therefore should be considered together.

Necessity of understanding video-informational processes specificity from the point of modern science (not heuristics), made forced to revise the fundamentals of information theory.
That's why appear a new paradigm of information's which based on fundamental analysis of information occurrence in the real World. Such new informational paradigm is a logical result of modern scientific knowledge development and allows switching from heuristic aspects to modern study the problem of information's quantity and quality joint evaluation. While studying this problem it was realized that artificial understanding the information of surrounding world is constitute as intellectual process. However, this process also is not to be intuitive and/or heuristic but it must be correct from the point of view of modern physical and mathematical fundamental science.

Thus, new approach to information based on informational paradigm of the World generalizes and supplements K. Shannon approach that based on heuristics of information's quantitative measurability, being stated long before the era of high information technologies even began. Traditional (by Shannon) informational approach has shown its efficiency at adequate informational tasks on information's quantity evaluation, but at the same time it turned out to be totally unhelpful when used for other purposes, i.e. at tasks where quality of information (geometry and logical properties of surrounding world) it the crucial factor. In other words, K. Shannon informational approach not allow finding out and/or taking into account quantitative and qualitative information's attributes that could be determined by corresponding physical (quantitative) and structural (qualitative) measurements.

In this connection, it is necessary to clarify the complicated issues of the real World's information content and respectively world's understanding is based on this information. The absence of answers on these issues makes it difficult to deep understanding of notions of information and its evaluation. In turn this circumstance prevents an effective development of high and ultra-high information technologies which includes an intellectual artificial (technical, computer, machine) vision.

In artificial vision (IV) the weak informational field is the carrier of weak video-information in the avail-able (in the hardware sense) video-information channel of one or another physical nature (Fig. 2) [2]. Therefore, the weak video informational field is so important for artificial vision. Exactly this physical field interacts with the system of vision and initiates the artificial vision where process the following interrelated tasks could be pointed out:

1. IV's direct problem assumes of evaluating of weak video-information by short-range interaction-measurement with vision system. The weak physical video-informational field is this interaction's intermediary to the physical component of video-receiver. The physical component of weak videoinformation's evaluation is a measure of such interaction, i.e. the weak video informational field's paraxial propagation and small aperture's of physical video-receiver allow to form the evaluation of weak video-information's physical component, traditionally considered as image.

2. IV's inverse problem provides a recovery for structure component of video-information which is weakened naturally as a result of transformation of video-information of common type to the weak one at the video informational field's propagation through the layer of space-time. Actually, the "objectvideo-receiver" space layer extent is traditionally considered at 
paraxial approximation [15]-[17] under conditions of material objects observation. It allows to register (in the near-field or far-field diffraction region) only the weak video informational fields (primary and/or secondary), which source is the corresponding weak video-information. Thus, being formed as a result of corresponding IV direct problem's solving the weak video-information evaluation (physical picture) initiates IV's inverse problem, which could be solved only on the base of long-range interaction-measurement evaluation of the weak video-information with specially formed video-information resources (video-thesaurus and video-intelligence) of vision

Physical effective Ryman’s space-time

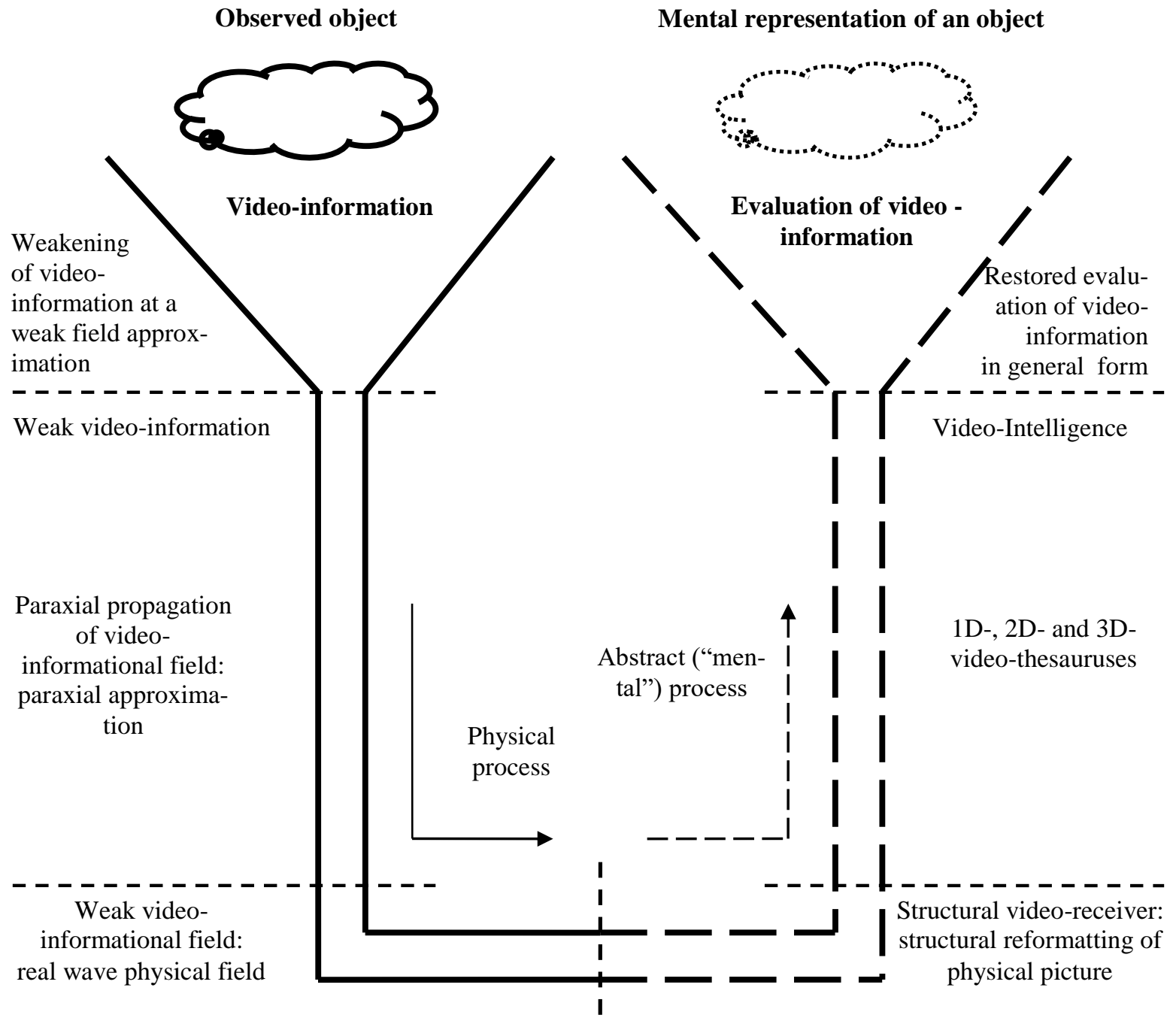

Physical video-receiver:

Building a physical picture system. These resources act as video-informational macroobjects that could be considered as measuring scales presented by a plenty of corresponding measuring video-structures. Long-range interaction is used without any intermediary's as physical field, what is the evidence of formal mathematical nature of such interaction and therefore is unlimited for quick speed, so in fact it is instantaneous. In practice, it could correspond to wide use of special tools to make the videoinformation's evaluation by formal methods of present-day mathematics, which is, as well known, represent an immense nonlinear structure with very strong interactions [18]-[20].

Virtual ("mental") space of AVIS

Mental representation of an object

information in general form

ideo-Intelligence

D-, 2D- and 3D-

ideo-thesauruses

Fig. 2. The conditional symbolic scheme of video-information weakening by paraxial physical channel of propagation, physical component's forming of the evaluation weak video-information and subsequent restoration of video-information in virtual ("mental") space of artificial vision intellectual system (AVIS). 
An evaluation of the weak video-information physical component in the form of physical picture should be considered at quantitative (physical) and qualitative (structural) aspects together, i.e. as partially physicalized. In this case a discrete physical reflection could be considered as plenty of physical samples with the properties of physical quants of videoinformation, which structure collapse to a point. Geometrical point as structure, as known, has a zero-dimensional order and thus it doesn't allow forming unambiguous higher order structures from the points. That is why, all geometrical elements (physical samples, pixels) reduced anyway to a point are unstructured. Using these elements, for example, it is possible to make a direct sample of field physical video-data on practice in the multi-element video-receiver mouth located in the lens's or reflector's focus (optical, thermal, acoustic, radio wave) [15][17], [21]-[23]. The example of indirect registration of physical video-data is a joint sample of amplitudes and phases of radioor acoustic holograms provided the further reconstruction of corresponding physical picture [24]-[26].

Structural (totally geometrized) component of the weak video-information evaluation or, in other words, structural reflection could be collated at qualitative (topology) aspect to the structure of the observed two-dimensional surface of visually controlled material object. As is known from topology, this surface could be divided into arbitrarily small parts ("be measured" with any high accuracy) by plenty of one-dimensional geometrical objects (lines) only [27]. Such division of twodimensional surface into the one-dimensional geometrical structures could be presented as its one-dimensional fibration.

In the surrounding macro world exactly are interactionsmeasurements (internal or external) of material systems generate the properties of any nature [1]. There is a single informational pattern in accordance which the interaction-measurement takes place only if the features of some dual interaction's components are comparable and one component's properties are macroscopic for the proper-ties of another one. The result of measuring, made by compare of micro-component (measured value) to macro-component (measuring scale) of corresponding dual interaction - measurement, could be a ratio and/or the system of ratio (structure that also includes video-structure) of this or that dimension. Meanwhile interaction-measurement macro-component could be presented as quantitative scale of ratios (dimensional physical ratios) and/or qualitative scale of spatial ratios systems (dimensional abstract video-structures).

There could be two types of measurements realized this way: physicals (quantitative) and structural (qualitative) [28][30]. Physical measurements mean the existence of quantitative (extensive) scales, e.g. absolute ones and ratios scales, expressed by numbers and allowed for calculations. Structural changes in its turn imply the existence of qualitative (intensive) scales, e.g., title and category scales dealing with the qualitative differences, when properties (attributes) ordering is possible, but calculations are not allowed, only comparisons. This is due to the fact that computing operations are applicable to spatial numbers, not to properties [30]. Moreover, in case of structural measurements there is no ability to set calibration and deviation (accuracy) for qualitative measuring scale, as the last one could be formed, e.g., as a list.
Physical quantities defined above as intensity measures of interaction-measurement of forms of matter (e.g., substances with appropriate field) naturally exist in nature. Therefore measuring of physical quantities assumes the existence of some physical device that made the interaction-measuring with the physical quantity and provides the result of measurement as some ratio (of number) of this or that physical dimension.

Structures in a natural way meetings in form temporary, spatial and space-time kinds of distribution (of laws of changes) of physical quantities. Structural interactionsmeasurements, aimed to obtain the assessment (of measuring result) of qualitative information, also assume the existence of some abstract (formally mathematical) instrument for structures "measuring". Such "measuring instrument" should be able to realize the interaction-measurement between the structures (ratios systems), providing the result of qualitative measurement in qualitative measure units, among which there could be simpler structures (in particular video-structures), which interpreted as dimensional structures.

It should be pointed out that AV process presented as a sequence of interactions-measurements (Fig. 2) is significantly oriented on the level of video-informational ratios systems (of video-structures), therefore concerning vision system it takes availability an advanced structural video-information resources on every level of structural video-information processing hierarchy. On the middle hierarchy's level (which is psychologically corresponding to perception and recognition of the weak video-structures), the support must be provided for the assessments (measurements) of those image's physical structures that took place in the composition of the physical picture. This is provided by $1 \mathrm{D}$ and 2D video-thesauruses. On the high and ultra-high levels of vision system hierarchy, psychologically corresponded to "objectification" (perception) and videoinformation's understanding, 2D video-information assessment initiates a video-information resource of high level (3D videothesaurus), which in turn initiates a video-information resource of ultra-high level of hierarchy (4D video-thesaurus or, in other words, video-intelligence) through $3 \mathrm{D}$ video-information assessment. All these video-information resources are presented as a video-experience, formed artificially and memorized by vision system as a result of her training (self-training) for 1D, 2D, 3D и 4D video-data, collated with the real world's objects that could be used as examples of possible objects for videoobservation.

As is known, thesaurus is presented as organized information having a number of various states, in this regard similar to entropy (although thesaurus and entropy have a different meaning) [31]. There-fore, thesaurus is measureable and measured in units of information. Thesaurus may be considered as internal informational resource of corresponding system, which could have a multilevel structure, e.g., hierarchically repeat at various level of detail, i.e. with the use of various types of morphism on every level of the hierarchy. Finally, in terms of systemology, thesaurus for the thesaurus itself could be considered as meta-thesaurus or, in other words, as consciousness or intelligence [31].

Thesaurus of vision system (video-thesaurus) is not observed in reality, as it is presented by passive video- 
informational macro-object (video-resource), which is able to make interactions-measurements with video-information on the high level of vision system hierarchy. For this, the videothesaurus has having in its composition advanced set of dimensional video-structures. Actually, video-thesaurus is a dynamic form of video-experience. In particular, video-experience of vision system in the conditions of cognitive interaction with real world gives a support to qualitative measurements on every level of hierarchy, which sequentially form of real world visual representation.

The forming and keeping of such video-informational macro-objects as video-thesaurus or video-intelligence justifies the existence of virtual space which is the "mental" space of vision system [32]. Exactly here is possible to get an abstract (formally mathematical) generation and association of videoinformation's evaluations, obtained on the all hierarchical levels of video-information's processing (evaluation). Virtual space of vision system is induced by information's evaluations in a manner similar to how video-information induces curved surrounding space-time. Thus, the virtual space of vision system could be presented as full and adequate reflection of the real world, as formal analogue of physical space-time. Such kind of virtual spaces could be considered as a type of "modeling reasoning", the essence of which are concluded in virtual reality modeling as phenomena in the field of machine psychology.

The concept of video-sensor space generates the concept of vision as of a "mental" vision for the real world's space-time content with help of video-experience definitely organized at video-sensor's memory. As J. Piaget wrote in this regard, «Looking at the object is an intellectual act already». Thus, natural and artificial vision could be collated psychical and intellectual mechanism respectively which will allow seeing the world as it is.

As is known, isomorphism of 2nd order between virtual ("mental") space-time and physical space-time is presented as psychologically reasonable assumption for explanation of the visual representations of the human. [33]. It is assumed that visual information contained at human's memory is in relation isomorphism of 2 nd order to the corresponding real video-data. In other words, the natural vision or artificial emulation "mental" images, formed in a mental space of corresponding vision system are resemblance to the real objects' images. Besides, a feature of this resemblance is that the ratio systems (videostructures), formed by mental images elements in the memory of vision system are the same as the ones between the elements of the video-informational images, formed by the real world. Thus, isomorphism of 2nd order differs from isomorphism in general (mathematical concept for designation of relationship for two objects basically identical to each other) in that it implies something closer to resemblance.

In this connection, one can put forward a hypothesis that artificial vision process, in case of being adequate its result, could be referred to video-information transformations, that don't change the physical condition of material object and don't make any influence on its observed characteristics. It is as a result of this is reaching effect of isomorphism of 2 nd order between video-information and its evaluation formed by vision (Fig.2). As is known, such transformations are called gauge ones [8], [34]. Gauge transformation of some quantity corresponds to arbitrary (scale) change of her value with constant gradients of this quantity being maintained. Absent change for the quantities observed at this transformation means gauge invariance of the real world observed [8]. In the human world gauge invariance is perceived as imperceptible game with numbers, which does not make visible changes to the observing world, therefore, people don't notice this transformation [34].

Based on video-information paradigm, can be implemented the natural decomposition of artificial vision as process of video-information evaluation, to some stages (sub processes) to simplify the solution of this definitely complex problem. For example, at the initial stage of artificial vision the interactionmeasurement of the weak video informational field with the video-receiver should be considered. It allows getting image which could be considered as evaluation weak videoinformation. This evaluation also has both physical and structural components for which could be designated according to their meaning where physical component of the image could be considered as physical picture, while the image's structural component present as structural picture [35].

On the further steps of artificial vision there is the processing of the image is realized, which is presented as 2D manifold (2-manifold) in terms of traditional geometrical intuition. At artificial vision the image processing aims to reconstruct the image from the weak (coarse) topology to the level of stronger (fine) topology typical for, e.g., 3D video-information evaluation. Such evaluation of video-information in view of the fact that with regard when 3D manifolds (3-manifolds) geometrical intuition doesn't work out will have a form of some abstract 3D image. The necessity of abstract 3-manifolds representation forces to abstractly consider the images that generate them (2manifolds). In case when space-time, which embraces solving artificial vision issue, accepted as four-dimensional one, abstract images in the form of 2-manifolds must totally give way to the ones in the form of 3-manifolds.

Even greater dimension of artificial vision task appears in case when controlled object is four dimensional, e.g. it takes form of space-time scene. In this case, the enveloping space is five-dimensional; therefore this circumstance can take one aside to the side from solving the problems of the real world's visual perception. Actually, additional (fifth) dimension is compactified and is enclosed with very small period $\left(10^{-31} \mathrm{~cm}\right)$, therefore 5-th coordinate is not observed [36], [37]. However, it should be taken in mind that artificial vision is generated by the effort to emulate the natural vision which is the sense organ; so, it is intended to fix a kind of projection of fourdimensional (space-time) processes on the really perceptible 3D (spatial) world. Modern researches in the area of small dimensions topology provide every reason to hope that the structure of four-dimensional space-time is defined by its 3D part's structure [18]-[20].

The forms of real objects in surrounding world could be rather complex so they are difficult to be described analytically and be modeled. The obvious solution, totally meeting the famous quotation "To understand is means to get used to and 
learn how to use" [R. Feynman], is in memorization of a plenty of real space-time ratio and systems of space-time ratios (video-structures). This is carried out to use their as dimensional ratios and video-structures, of which as a result of their association (during training and self-learning) the video informational macro-objects (qualitative measuring scales) of vision system could be formed.

\section{Structural AND FunCTIONAL FEATURES OF INTELLIGENT SYSTEMS OF ARTIFICIAL VISION}

Artificial vision intelligent system (AVIS) solves the following problems:

1) Evaluation of weak video-information about of the visual observable condition of the real world's objects as some of $1 \mathrm{D}$ and $2 \mathrm{D}$ images. These images correspond to relatively "coarse" topologies of 1- and 2-manifolds, which could be collated with the intuitive understandable images of lines and surfaces.

2) Evaluation of "strong" video-information in the form of 3D and 4D images of observed objects of surrounding world, those are carriers of more "thin" topology. These images are matched 3- and 4-manifolds which are representing intuitionally non-understandable images.

Generally speaking, these two global video-information processing (evaluation) stages assume a significantly advanced set of complex and non-trivial tasks making hard for perception of important de-tails of artificial vision process. Therefore, it is necessary to develop a process decomposition of the global stages to sub-stages with shallower local subtasks. Their understood on easier when considering the process of videoinformation evaluation at AVIS as an intellectual multistep technology of video-information evaluation.

Structurally-functional scheme of AVIS stages is presented on Fig. 1. The stages of video-information's and video informational field's forming were studied at works [1]-[4], so it is not necessary to add any comments. Then by processing of weak video- informational field physical video-receiver of vision system formed physical video-data is as a plenty structureless samples of physical picture. Structural video-receiver of vision system form a structural video-data in the form of plenty of elementary structural samples (local qualts) by physical video-data's reformatting.

Physical picture is formed by video-receiver's physical component (television camera, thermal imaging, multielement radio- or hydro acoustic antenna) that provides a physical image's registration as a space-time sample of physical video-data (optical, thermal, radio wave and ultrasound). The sizes and number of these samples are defined by resolution of videoreceiver as well as transverse and longitudinal (according to depth) dimensions of its field of vision. The importance to provide high resolution for the depth on the initial stage of artificial vision is confirmed by known experiments with randompointed stereograms [21], [38]. As can be seen from these experiences, volumetric (stereoscopic, holographic) vision could not only precede the shape's perception, but also carry out without her recognition. In other words, perception of outlines and forms is not a prerequisite for achieving a volume effect, i.e. "not so much the contours are push [us] on the thought of depth, how much a depth - on the thought of contours" [J. R. Shiffman].

\section{INFORMATIONAL HYPERGRAPHS OF VIDEO- INTELLIGENCE AND STRONG ARTIFICIAL INTELLIGENCE}

In present time, the gauge theory at the solving some field problems successfully goes beyond a compensatory wording that which has proved itself well at in case of internal symmetries. Therefore, modern gauge approach could be formed not only proceeding to local invariance principle but also within the framework of general description classical fields on the base of fibration formalism which is commonly used at the transition from the local description of solved problem to its global one [7]-[9], [39].

The role of geometrical methods in various areas of science and technology increased significantly now. As is known, mathematical analysis methods provide a qualitative phenomena's characteristic in small values of changes of these or that parameters. Geometrical methods, in its turn, provide to a qualitative description of the same phenomena at large values of changes of its parameters. The other equally important peculiarity of geometrical methods is the convenience of geometric language for various regularities explaining. In this regard along with mathematical analysis of manifolds (with the use of modern differential geometry) a fibrations theory which is turned out to be the most convenient one at many applications has been developed greatly.

Already at the first stages gauge interpretation of videoinformation faces difficulties as generally gauge fields is connectivity as well as video informational field is a metric field (In this case two conditions are imposed on the connectivity: of symmetry and metricity [7], [8], [25], [40], [41]). The gauge status of video-informational field remains unclear but is sufficient productive in compensatory scheme of gauge theory in case internal symmetries. This allows to in particular correctly identify the peculiarities of video-information interaction generating the video-information and her internal structure's which from the help of coordinate transformations only intuitionally are define. However, the existing experience forming of gauge theories for field systems indicates that for the space-time symmetries the compensatory wording of gauge theory is turned out to be not effective enough so it is necessary to use fibrations mathematics [7]-[9].

At the initial stage of artificial vision equations forming, the traditional compensatory wording of gauge theory is quite enough. That is why, the hypothesis advanced earlier, asserting that the objects in effective Riemannian space-time and virtual ("mental") space of AVIS are connected by gauge transformation, should be used. This hypothesis is correct due to the fact that, as is known, gauge transformation not reflected on the observed state (physical and structural) of the a real world's objects [8], [34]. In this case, there is every reason to assume that video-information about a spatial content of the real world and its evaluation in mental space of AVIS to be connected with each other by isomorphism of 2 nd order, which implies something closer to the similarity than isomorphism [33]. In other words, the video-information evaluation process is a vision process, in case there a relations between the natural vid- 
eo-information in a physical space-time and its evaluation in "mental" space of AVIS is close to of equality relations.

Limited opportunities of classical informational approach hardly met science and technology needs already at the end of 20th century. However currently (as established and shown in the works $[1,2])$ quantitative (physical) and qualitative (structural) components of information should be considered together. Wherein, in the expression for a full video-information $t_{m}^{n}$, considering at coordinates of Minkowski space it's physical $t_{m(E)}^{n}$ and structural $t_{m(S)}^{n}$ components enter additively making it's share in a full video-information. In general it could be presented as

$t_{m}^{n}=t_{m(E)}^{n}+t_{m(S)}^{n}$.

However, the surrounding material world due to nonlinearity of corresponding sensors having usually quadratic characteristic of sensitivity's is usually percepted on full information intensity (power). In doing so, the expression for full video-information intensity $I_{m}^{n}$ could be quite obviously presented as

$I_{m}^{n}=\left(t_{m}^{n}\right)^{2}=\left(t_{m(E)}^{n}+t_{m(S)}^{n}\right)^{2}=\left(t_{m(E)}^{n}\right)^{2}+2 t_{m(E)}^{n} t_{m(S)}^{n}+\left(t_{m(S)}^{n}\right)^{2}$

As shown from the latest expression, the one for videoinformation intensity in common form allows to explicitly identify its components interaction carried out by their multiplying. This confirms the assertion developed in works [1, 2] that the strong interaction of video-information components is possible only when each of them is dual. Namely, physical component of video-information is only partly physicalized and structural one is only partly geometrized respectively. Precisely because of duality for strongly interacted components a video-information becomes a system of physical and structural components considered together.

Video-information components interaction is particularly evident in the expression for weak video-information intensity

$$
\begin{aligned}
& \stackrel{(0)}{I_{m}^{n}}=\left(\begin{array}{l}
(0) \\
t_{m}^{n}
\end{array}\right)^{2}=\left(\begin{array}{ll}
(0) & (0) \\
t_{m(E)}^{n} & +t_{m(S)}^{n}
\end{array}\right)^{2}=\left(\begin{array}{l}
(0) \\
t_{m(E)}^{n}
\end{array}\right)_{\rightarrow 0}^{2}+2 \stackrel{(0)}{t_{m(E)}^{n}} \stackrel{(0)}{t_{m(S)}^{n}}+ \\
& +\left(\begin{array}{l}
(0) \\
t_{m(S)}^{n}
\end{array}\right)_{\rightarrow 0}^{2} \rightarrow 2 \stackrel{(0)}{ } \quad \stackrel{(0)}{t_{m(E)}^{n}} t_{m(S)}^{n}
\end{aligned}
$$

Where $t_{m}^{n}$ - weak video-information.

The meaning of the expression (3) is that in case of weak video-information the interaction of its physical and structural components is realize by its multiplying.

Let us consider the main aspects of the idea on which video-information evaluation in AVIS is based. As videoinformation evaluation is a video-information also, it is obvious that the results obtained above about video-information structure are also right for video-information evaluation $\hat{t}_{m}^{n}$, which is generally presented as

$\hat{t_{m}^{n}}=\hat{t_{m(S)}^{n}}+\hat{t}_{m(E)}^{n}$

The surrounding material world due to video-sensors nonlinearity is commonly percepted by video-information intensity (power). Therefore, the expression for the intensity of videoinformation evaluation could be presented as

$$
\begin{aligned}
& \hat{I_{m}^{n}}=\left(\hat{t_{m}^{n}}\right)^{2}=\left(\hat{t_{m(S)}^{n}+\hat{t}_{m(E)}^{n}}\right)^{2}=\hat{I}_{m(=)}^{n}+\hat{I}_{m(\times)}^{n}, \\
& \hat{I}_{m(=)}^{n}=\left(\hat{t_{m(S)}^{n}}\right)^{2}+\left(\hat{t_{m(E)}^{n}}\right)^{2}, \hat{I_{m(\times)}^{n}}=2 \hat{t}_{m(S)}^{n} \hat{t}_{m(E)}^{n},
\end{aligned}
$$

Where

$$
\hat{t_{m}^{n}}, \hat{t_{m(S)}^{n}}, \hat{t_{m(E)}^{n}}-\text { accordingly, video-information evaluation, }
$$
its structural and physical components;

$\hat{I_{m}^{n}}, \hat{I}_{m(=)}^{n}, \hat{I}_{m(\times)}^{n}-$ accordingly, intensity of video-information evaluation, its background component and the wanted component, which considered as multiplication of physical and structural components of video-information assessment.

From the expressions (5) and (6) it follows that videoinformation evaluation intensity includes its physical and structural components interaction carried out by their multiplying.

Multiplicative character of components interaction of evaluation weak video-information is particularly demonstrable in the expression for the intensity of weak video-information evaluation

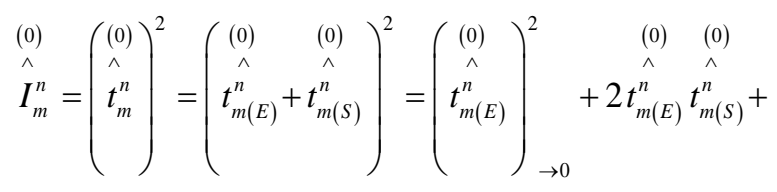

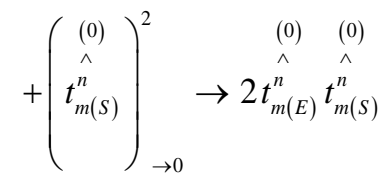

or

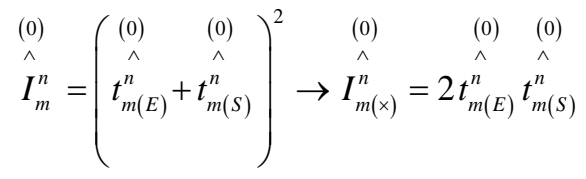

Currently when researching artificial vision systems there are the attempts to intuitionally circumvent the laws of nature, e.g. with the help of all kinds of neural networks, which is able to react only to the physical component's intensity of evaluation weak video-information collated to traditional physical 
image. By "deep" training or some other heuristic method on the base of neural networks, the researchers try to solve the problem of artificial vision with the structural component not being taken into consideration. However, according to expression (8) in this case inadequate evaluation weak videoinformation is formed.

In the process of solving the tasks of intellectual artificial vision a set of hierarchical video-information evaluations could be considered as a result. Its psychological analogues are recognition, perception and understanding of videoinformation. Such complex evaluation of video-information allows for every image of the real world to collate "mentally" the corresponding abstract image depending on the depth of solving video-information processing (evaluation) problem.

The system-forming factor of AVIS is upward videothesauruses hierarchy, including ultra-high level's videothesaurus or otherwise video-intelligence. The whole this hierarchy is functioning in full compliance with post Shannon information paradigm as internal video-informational macroobjects and provide the strong video-informational interactions on every hierarchy's level.

On the initial stage of artificial vision due to the relatively small apertures dimensions of video-receiver the space-time layer of «observable object - video-receiver» relative to videoinformational field acts as a narrowband spatial filter. Therefore, the strong initial video informational field (secondary or primary), whose source present video-information with strong (thin) topology transforms into weak video-informational field obtained by narrowband filtering by specified spatial layer (Fig. 2). Weak video-information can be considered as the source of such weak video informational field, which formed from general view of video-information by its relative topology weakening (coarsening). Weak video informational field initiates artificial vision process by activating of passive video informational resource of AVIS. In doing so, physical and structural video-receivers of the system both provide a full sample of video-data, presented as physical quanta (structureless samples) and structural quanta (local qualts) arrays is considered together.

Fibration space as is known could be considered as some $k$-dimensional space (base), which points are changed to $l-$ dimensional spaces (layers) with its own geometry [8]. Therefore, 3-manifold evaluation in the form of fibration could be considered as set-theoretic sum of two-dimensional layers. At computer representation this set theoretically sum (taking into account idempotency law where logical structures coefficients and exponents are eliminated from consideration) could always be reduced to the form of disjunctive union of layers parameterized by the database and "bonded" together by fibration space topology [8].

The majority of the real world's objects have a videoinformation, which due to its complexity and unpredictability in general case could not be described formally in details. Therefore, there is a necessary to act according to the modern information template instead of trying to describe the real world formally. According to this template, any process that generates information at this or that form or providing its eval- uation should be organized as interaction-measurement. This implies the presence in corresponding video-information system (physical or abstract) having at least two collated and strongly interacted micro- and macro-component. Microcomponent of interaction is as measurable value while macrocomponent acts like a measuring scale "calibrated" at corresponding dimensional values or dimensional structures in order to quantitatively and/or qualitatively be evaluation results this interaction-measurement.

At such approach the process of artificial vision could be considered as sequential empirical process of hierarchical measuring scale forming for video-structures (similar to multilevel Bourbaki scale) and its further "mental" use for videoinformation evaluation about the spatial content of the real world. By the way, it was introduction of the Bourbaki scale formal concept has allowed N. Bourbaki formally introduce the concept of structure.

Let us consider the features of sequential forming for some video-thesaurus as video informational macro-object ordered by the principle of accounting for all paired relations between "dimensional" video-information's that thesaurus consists of. This forms a certain systems of relations (structure) of videothesaurus. Actually, for the composition of two videoinformation in general form, when there is no loss of videoinformation, their pairing is the ratio of union (of logical sum) is the simplest way. As is known, ratio could be represented generally as some operation not only between the numbers but also between the objects of any nature including the one between the structures. For the number of video-information's more than two (three, four, etc.) one could speak number of elements $\left(r_{1}\right)$ of video-thesaurus which at the increase of their number $\left(r_{1}=3,4,5, \ldots K\right)$ is bound together in as much paired relations as different combinations from $r_{1}$ elements for two could be formed. It allows to present video-thesaurus forming scheme as $r_{1}\left(r_{1}-1\right) / 2$ combinations paired relations of «dimensional» video-information (Fig. 3). This scheme provides video-thesaurus forming as a set-theoretic sum of informational components of the same dimension that could be transformed in accordance to idempotency law to more convenient (for computer analysis and synthesis) form of disjunctive union of these components. Thus, informational meaning of fibration and disjunctive union for of the components of having the same dimension mean that in geometrical structure is introduced structure of logic and this so it allows logically analyzing and synthesizing the relevant geometry.

The expression (9) as applied to 3D video-thesaurus demonstrates the transition from set-theoretical form of videothesaurus to its representation in accordance with idempotency law in the form of disjunctive union of its video-informational components of the same dimension which appropriate to the high level of AVIS hierarchy

$$
\theta_{m}^{n}=(K-1) \sum_{\eta=1}^{K} \hat{t_{m(\eta)}^{n}} \stackrel{\text { idempotency law }}{\Rightarrow} \bigcup_{\eta} t_{m(\eta)}^{n}, \quad \eta=\overline{1, K}
$$

where 
a)

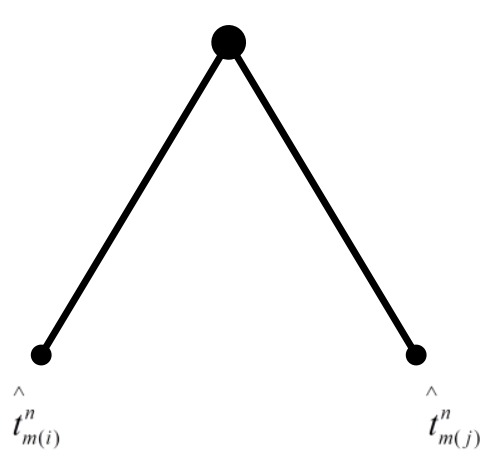

$$
\begin{gathered}
\theta_{m\left(r_{1}\right)}^{n}=\sum_{\eta=1}^{2} t_{(\eta)}^{m n} \\
\eta=1,2, r_{1}=1
\end{gathered}
$$

c)

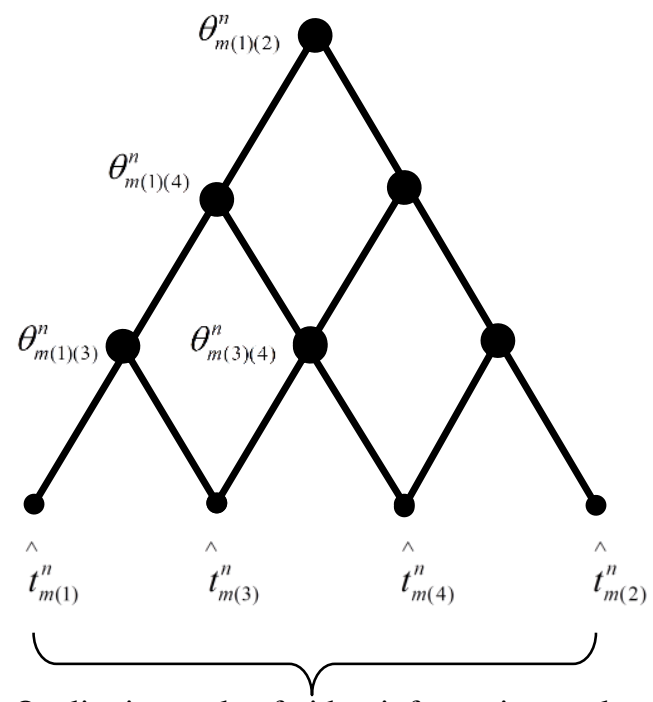

Qualitative scale of video-information evaluations (video-thesaurus)

$$
\theta_{m(1)(2)}^{n}
$$

b)

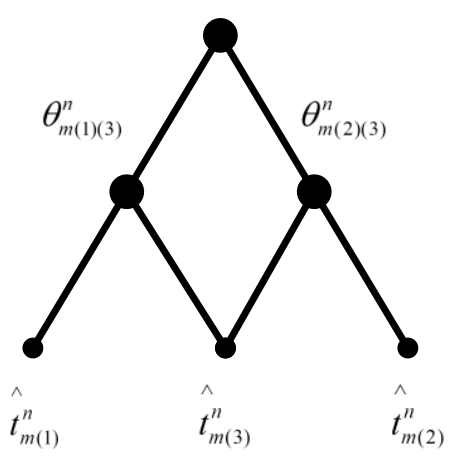

$$
\begin{aligned}
& \theta_{m\left(r_{1}\right)}^{n}=2 \sum_{\eta=1}^{3} \hat{t}_{m(\eta)}^{n} \\
& \eta=\overline{1,3}, r_{1}=3
\end{aligned}
$$

$$
\begin{aligned}
& \theta_{m\left(r_{1}\right)}^{n}=3 \sum_{\eta=1}^{4} \hat{t}_{m(\eta)}^{n} \\
& \eta=\overline{1,4}, \quad r_{1}=6
\end{aligned}
$$

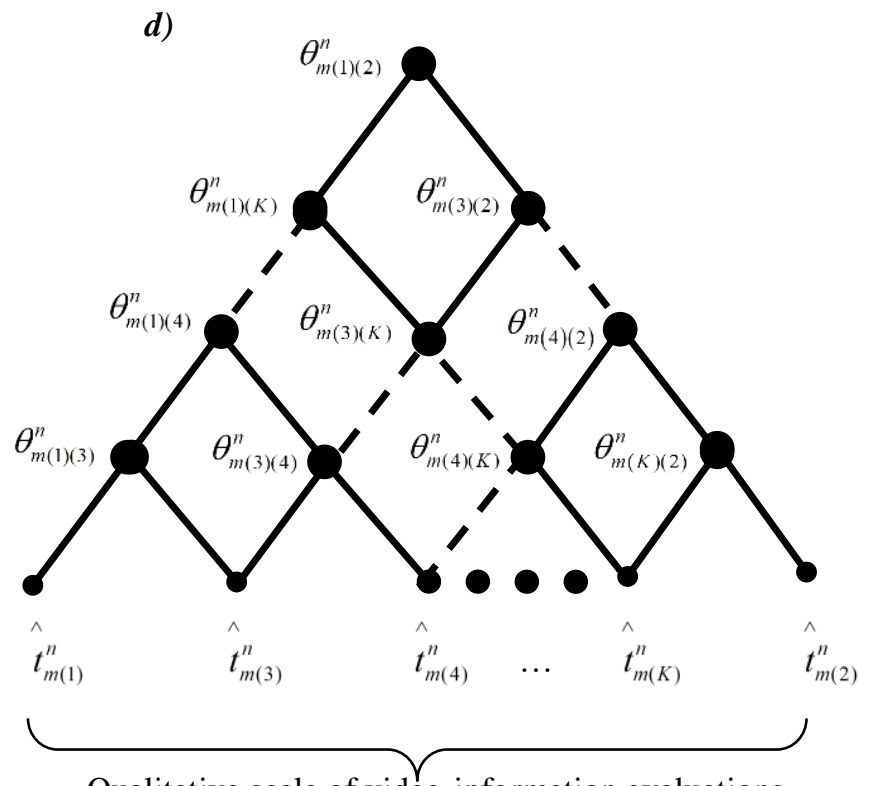

Qualitative scale of video-information evaluations (an advanced video-thesaurus)

$$
\begin{aligned}
& \theta_{m\left(r_{1}\right)}^{n}=(K-1) \sum_{\eta=1}^{K} \hat{t}_{m(\eta)}^{n} \\
& \eta=\overline{1, K}, r_{1}=K(K-1) / 2
\end{aligned}
$$

Fig. 3. Informational hypergraphs of video-thesauruses $\theta_{m}^{n}$ of 3-th (b), 4-th (c) and K-th (d) ranks with a heterarchicat organizational structure on the base of paired relations of $\theta_{m(i)(j)}^{n}(a)$ video-information evaluations.

$\theta_{m}^{n}-3 \mathrm{D}$ video-thesaurus: considered as 3D video informational macro-object or qualitative measuring scale representing the union of "dimensional" video-information of the same spatial dimension; 


$$
\hat{t_{m}^{n}}=\bigcup_{\rho} \hat{t_{m(\rho)}^{n}}, \rho=\overline{1, R} \text { - current "dimensional" video- }
$$

information in the form of abstract 3D image (of a threedimensional manifold), which fibration into two-dimensional images of the observing object's;

$K$ - video-thesaurus rank; $R$ - rank of a three-dimensional manifold.

Video-thesaurus development schemes are shown in Fig. 3, presenting hypergraphs, where assigned relations don't have fixed parity. The edges of hypergraph connect not the single pairs of vertex, but rather their subsets. It is important to emphasize that information contained at hypergraph is non-metric information and could be characterized only topologically. Hypergraphs, shown on Fig.3 correspond to the process of video-thesaurus sequential development as of qualitative "measuring" scale.

Generally, any video-thesaurus be formed by aplenty of video-experiences as sequentially increasing array of videoinformation evaluations. This array of video-information evaluations units all the paired relations between them and present itself as video-informational macro-object of some K-th rank. The rank of such macro-object corresponds quantity to its par-

a)

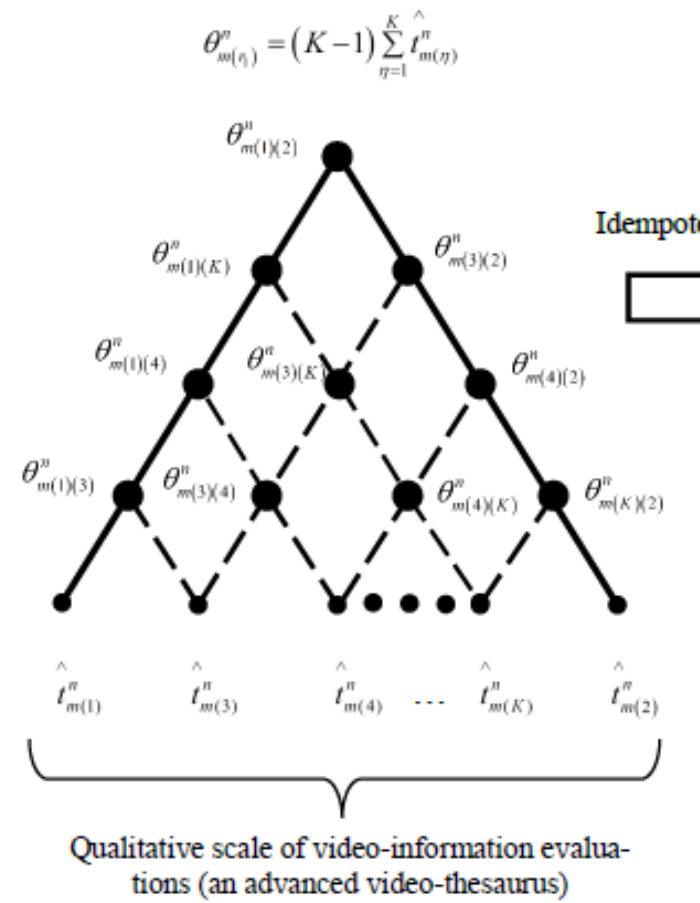

tions (an advanced video-thesaurus) tial components (of "dimensional" video-informations) covered by the heterarchy of paired relations. This heterarchical organization structure of video-thesaurus that must form during AVIS training, generally speaking, very difficult at implement (Fig. 3). However, in accordance to idempotency law such integration of "dimensional" video-information, pairwise organized combinatorically, degenerates into much more simplier empirical qualitative measuring scale of video-structures in the form generalized informational hypergraph, which quite easy to be realized (Fig. 4).

The theory of physical structures currently proves that there does not exist nontrivial theories of ternary, tetradic, etc. systems of relations. In other words, nature limited itself only to cases of binary and unary systems of relations [36], [42]. Thus, the considered variant of video-thesaurus development at video-experience accumulation by combinatorial union of all paired additive compositions of video-information assessments is turns out to be the only possible one.

It should be noted, that in this case rather important additive law of composition acquires geometrophysical interpretation. Actually, unlike elementary algebra, additive representation of law of composition does not necessarily mean addition [43].

b)

$$
\theta_{\mathrm{m}\left(\eta_{1}\right)}^{\prime \prime}=\bigcup_{\eta}^{\prime \prime} t_{m(g)}^{\prime \prime}
$$

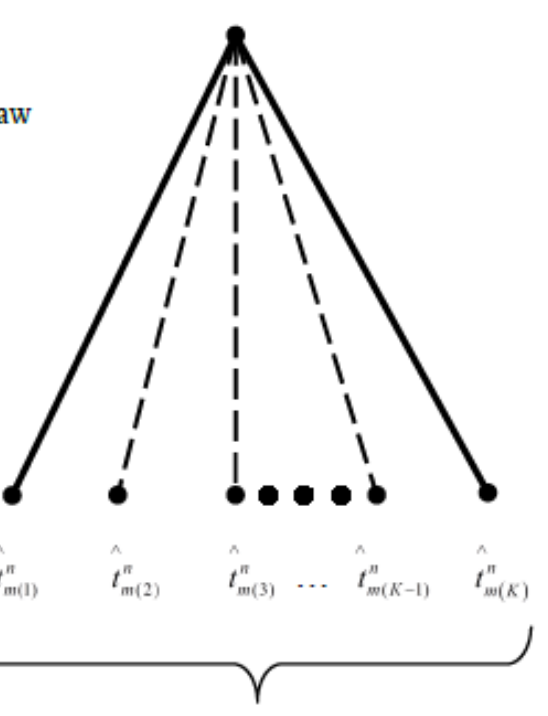

Qualitative scale of video-information evaluations (an advanced video-thesaurus)

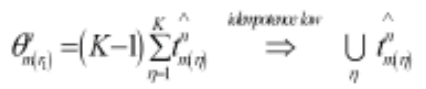

$$
\begin{aligned}
& \eta=\overline{1, K}, r_{1}=K(K-1) / 2
\end{aligned}
$$

Fig. 4. Transformation of informational hypergraph video-thesaurus as set-theoretic sum of video-information evaluations (a) in to much more simple and available structure (b) of generalized informational hypergraph. 
This operation only seemingly looks like an ordinary addition operation, but essentially, it is a disjunctive union of video-informations evaluations on corresponding (low, middle, high or ultra-high) hierarchy levels in ATIS.

From the expression (9) follows what 3D videothesaurus in accordance with idempotency law and 3D generic hypergraphs structure becomes of qualitative measuring "scale" for 3D video-informatios. These "dimensional" video-

informations are presented by abstract images $\hat{t}_{m}^{n}$, collated to the $3 \mathrm{D}$ video-information assessments and fibered into congru(0)

ent two-dimensional images $\hat{t}_{m(p)}^{n}$, which correspond to weak video-information evaluations. Here it should be taken into account that dimension of some set (manifold) means its decomposition on as small as possible by the subsets (fibers) of dimension $n-1$ and the impossibility of such split by subsets (fibers) of dimension $n-2$ [27]. Therefore, the 3D videoinformation evaluation formally could be presented as

$\hat{t}_{m}^{n}=(P-1) \sum_{p=1}^{P} \hat{t_{m(p)}^{n}} \Rightarrow \stackrel{(0)}{\hat{\bigcup}} t_{p}^{n} t_{m(p)}^{n}, \quad p=\overline{1, P}$

Where

$\hat{t}_{m}^{n}-3 \mathrm{D}$ video-information evaluation presented by fibration on evaluations congruent $2 \mathrm{D}$ video-information;

(0) $\quad(0)$

$\hat{t_{m(p)}^{n}}=\hat{t_{(p)}^{m n}}$ - current two-dimensional layer as one of evaluations congruent $2 \mathrm{D}$ video-information;

$P$ - number of two-dimensional layers (rank) fibration space 3D video-information .

According to the expression (10) video-information evaluation is presented as manifold in the form of disjunctive union (0) (0)

of fibers $\hat{t}_{m(p)}^{n}=\hat{t}_{(p)}^{m n} \quad$ (in case of the evaluation weak videoinformation there is no need to distinguish co- and contravariant tensor components), forming its atlas [8], [9], [13], [27], [39].

Taking into account this fact, the current 2D fiber in the (0)

form of $2 \mathrm{D}$ evaluation weak video-information $\hat{t}_{(p)}^{m n}$ could be presented splitting to $1 \mathrm{D}$ evaluation video-information and therefore could take the form of

$$
\hat{t_{(p)}^{m n}}=(Q-1) \sum_{q=1}^{Q} \hat{t_{(q)}^{m n}} \Rightarrow \bigcup_{q} \hat{t_{(q)}^{m n}} \quad, \quad q=\overline{1, Q}
$$

Where

$$
\hat{t_{(q)}^{m n}} \text { - evaluation weak 1D video-information; }
$$

$Q$ - quantity of one-dimensional layers (rank) of 2D videoinformation evaluation.

In its turn, each $1 \mathrm{D}$ video-information evaluation present as one-dimensional geometrical object (one-manifold), which could be presented as linear combination of elementary samples (quanta) of physical and structural video-data, considered together

$\hat{t}_{(q)}^{m n}=(L-1) \sum_{l=1}^{L} \hat{t_{\times(l)}^{m n}} \Rightarrow \bigcup_{l} \hat{t}_{\times(l)}^{m n}, \quad l=\overline{1, L}$

Where

$$
\hat{t_{\times(l)}^{m n}}=\bigcup_{r} d\left\{\begin{array}{c}
(0000) \\
\hat{t_{E(r)}^{m n}}
\end{array}\right\} \hat{t_{E(r)}^{m n}}, r=\overline{1, R} \text { - full video-data in the }
$$

form of elementary physical and structural samples considered together;

$L$ - quantity of components (rank) of 1D evaluation videoinformation;

$(0000)$

$\hat{t_{E(r)}^{m n}}, r=\overline{1, R}-$ structureless sample of physical video-data;

$d$ - operator of differentiation.

Full sample of elementary video-data is presented as indivisible joint physical and structural video-information quantum underlying the base of video-information evaluations hierarchy (Fig. 5). This element is fundamental to provide an intellectual process of video-information evaluations forming and on the importance can be compared only with the string that is considered by modern physics as fundamental element of matter [9], [44].

Actually, at intellectual artificial vision based on AVIS video-informational processes are the processes of sequential formation of video-information evaluations upward hierarchy. In these processes evaluations of structural components of videoinformation are dominated, as their nature provide the technology of intellectual assessment for video-information. The physical components evaluations play an important supportive role to avoid video-information loss during its evaluation.

It should be noted, that local qualts (structure quanta) be formed action by differentiation operator's on the structureless sample of physical picture. Only in case of weak videoinformation outer differentiation operator acts like a common differentiation operator in the vicinity of the point coinciding with spatial coordinates of corresponding structureless sample on the physical picture

$$
\hat{t_{S(l)}^{m n}}=d\left\{\begin{array}{c}
(0000) \\
t_{E(r)}^{m n}
\end{array}\right\}, r=\overline{1, R}
$$

Where 
$(0000)$

$\hat{t_{E(r)}^{m n}} \quad$ - structureless sample of physical picture;

$R$ - rank of the differentiation operator's "window" of physical picture.

The expression (13) refers to the low level of AVIS hierarchy and corresponds to the reformatting of structureless samples of physical picture into the local qualts.

Using the above formalism as a guiding principle can be built generic hypergraph of strong artificial intelligence in the form of a composition of interacted artificial intelligences of sensor and non-sensor nature (Fig. 5). Strong artificial intelligence of sensor nature can be considered in the form of a fibration on video-intelligence (artificial vision), audio-intelligence (artificial hearing), tactile intelligence (artificial touch), chemo intelligence (artificial smell) and many other artificial intelligences that use the available variety of sensors of physical nature. The strong artificial intelligence of non-sensor (structural, abstract) nature is a much more numerous ("broad"). It can be considered as a union of mathematical, physical, musical, engineering, driving, household and many other kinds of intelligences constructed (trained) on the basis of relevant structural (abstract) information. All these numerous partial intelligences are considered together, what allows to present strong artificial intelligence as

$\chi_{m}^{n}=\alpha_{m}^{n} \circ \beta_{m}^{n}$

Where

$\chi_{m}^{n}=\alpha_{m}^{n} \circ \beta_{m}^{n}-$ strong artificial intelligence in whole is formed as a composition partial strong artificial intelligences (of sensor and non-sensor nature considered together) of the brain-like system;

- - mathematical symbol of composition;

$\alpha_{m}^{n}-$ partial strong artificial intelligence of sensor nature of rank $N$ (including video-intelligence $\alpha_{m(3)}^{n}$, see Fig.5)

$\alpha_{m}^{n}=\bigcup_{z} \alpha_{m(z)}^{n}, \quad z=\overline{1, N}$

$\beta_{m}^{n}-$ partial strong artificial intelligence of non-sensor nature of rank $M$

$\beta_{m}^{m}=\bigcup_{s} \beta_{m(s)}^{n}, s=\overline{1, M}$.

Informational approach to the strong artificial intelligence as to an informational category allows to consider uniformly its numerous partial intelligences of any nature united by its common attribute - information, as the information is a measure of strong interaction (interaction-measurement) comparable forms of matter. In doing so, the interacted forms of matter, e.g., physical (chemical) and structural (abstract) ones or, in a more general form, quantitative and qualitative ones should always be considered together. An abstract (image) thinking needed at complex and dynamically changing envi- ronment to assess the current and prospective state of the surrounding world underlies the strong artificial intelligence [45 48]. Strong artificial intelligence in a brain-like system acts as a backbone system-forming informational macro-object in the form of some complex qualitative measuring scale to obtain qualitative measurements on the ultra-high level of the system's hierarchy. This provides building a brain-like system for a numerous qualitative joint evaluations for informations of any nature which as a result with the need formed an understanding of the surrounding real World

On Fig. 5 is shown a generic hypergraph of strong artificial intelligence, in which one of artificial intelligences, namely, the video-intelligence, is shown with the unfolded internal structure of the ascending hierarchy of its evaluations of videoinformation. The initial fundamental feature of information, that the quantitative and qualitative information components are always considered together, is used when forming the hypergraph. It corresponds to the concept of a new (post Shannon) information approach under which the paradigm of information is united and dos not depend on of the nature of components for corresponding interactions-measurements which rank could be extremely large. Thus, not only natural advanced biological systems, but also technologically developed artificial ones (that could be formed as intelligent systems of different purposes) can have intelligence. Currently such systems most often implement intuition ways intellectual processing (evaluation) of information that have a heuristic nature and could be formed, e.g., on the base of various neural networks. However, times of "intelligent systems", which work could not often be explained even by its researcher, are passing. They must be replaced by truly intellectual brain-like systems adequate to intellect's complex nature that could be understood only by the use of current advances in basic science. This circumstance tightens the requirements for the educational level for of modern intelligent systems researchers, who should use fundamental science not as a philosophy, but as a necessary working tool.

Natural and artificial intellects the highest achievements of biological evolution and technological progress in the field of data processing systems. Moreover, in both cases it is the vision system, provided an adequate abstract world's reflection in virtual (mental) space of system, which generates an abstract thinking as high-effective data processing technology. An abstract thinking becomes the determining factor of natural intellect's evolution, that generated its pursuit of self-knowledge, by the creation of emulator an artificial intelligence including. However, an abstract thinking also underlies the artificial intellect technology. Therefore, an artificial intelligence could have unlimited evolutionary possibilities by preliminary training and a further self-training that would generate the new knowledge for all or separate areas of science. Furthermore, an advanced artificial intelligence is certainly able to effectively support a natural intellect (e.g. as a brain-like supercomputer) when solving critical tasks for humanity. In doing so, its simplified problem-driven versions could provide on a competitive basis an effective alternative to natural intellect when solving numerous secondary tasks from the society, industrial production and the surrounding material world. 


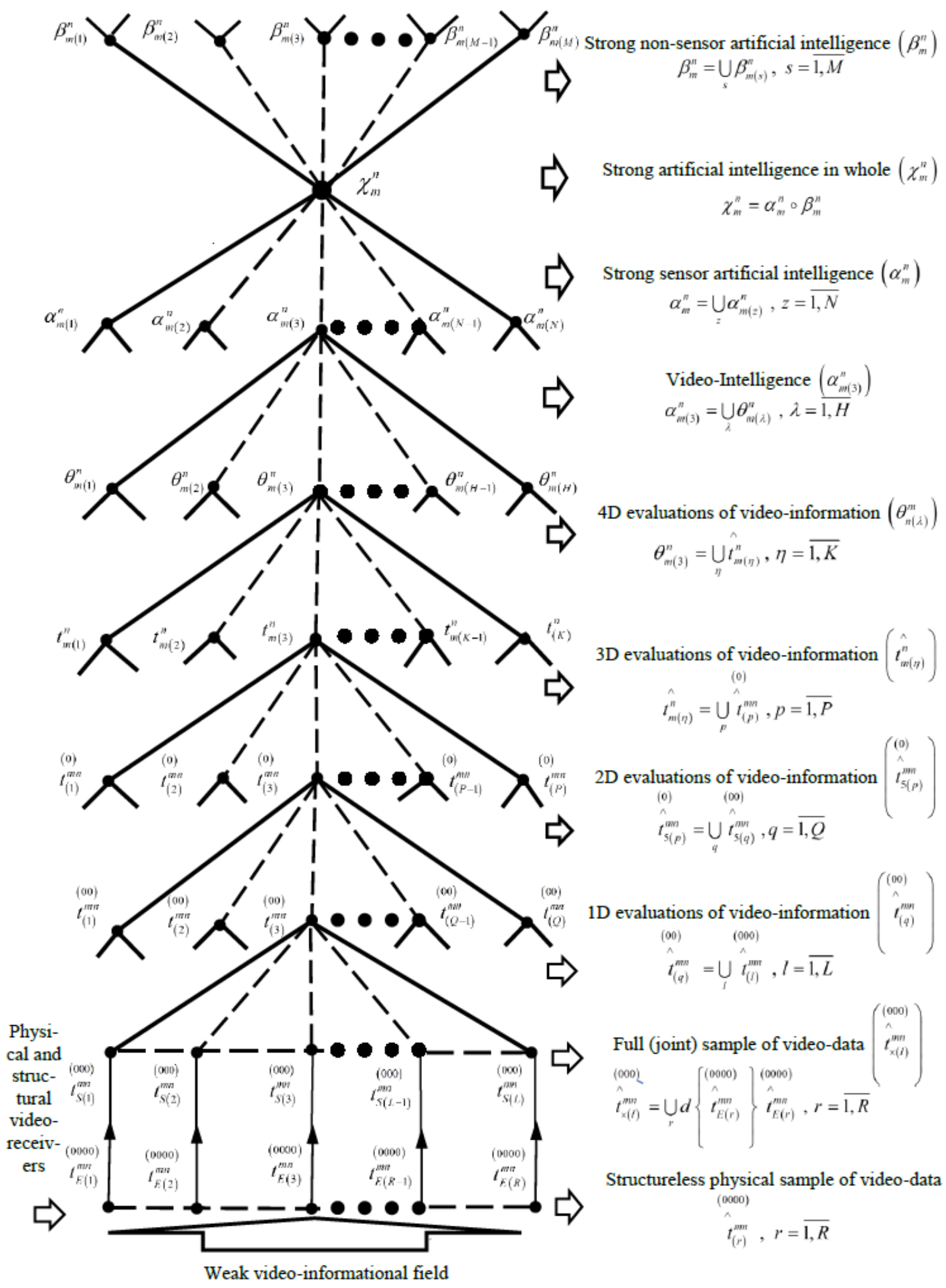

Fig. 5. Strong artificial intelligence's hypergraph in whole are regarded a composition the generalized hypergraphs of it non-sensory and sensory components in which as an example of a deeper structures being considered video-intelligence as hierarchy generalized hypergraphs of evaluations video-informations and videodata. 


\section{DISCUSSION}

This article discusses the development of post-Shannon informational approach, created at "NeocorTek Lab" where this approaches was additionally adapted to the problem of videoinformation evaluation in artificial vision. NeocorTek Lab suggested of the productive informational approach-synthesis for creation of applications on the basis of artificial intelligence systems (including strong artificial one). Created approach (at the absolute abstraction from the concepts and technologies of neural networks) is self-contained and is able to provide an effective support to the processes of forming the truly intelligent systems for various applications. Such intelligent systems have structure that comparable to the structure of the human's neocortex and therefore they can be considered as brain-like systems.

Artificial intelligence, in our view, could be considered as an intelligence systems ability (e.g. of artificial vision similar to human's vision) to the forming of virtual "mental" spacetime where all the information about the surrounding world could be adequately presented in the form of comparable and interacted "mental" formally images. Such approach allows to correctly setting and adequately solving the tasks of understanding concerning information.

Currently the systems of socalled "weak" artificial intelligence mainly aimed to solve the recognition tasks and based on neural networks technologies (deep and many others) are already quite widespread. According to the current informational approach-synthesis, these heuristic technologies do not correspond to the informational essence of intellectual mechanisms of recognition, perception and understanding of information. Therefore, informational approach at these systems almost does not work, especially as much critically important information is irretrievably lost at the entrance area of neural network already. The further compensation these fundamental losses of information by any heuristic method are impossible.

The main achievement of NeocorTek Lab which located in Russia is the creation of productive information theory of artificial intelligence which allows creating intelligence systems meaningfully and purposefully. Initial elements of this theory were demonstrated in the current article. In the process of indepth study of artificial vision managed to open completely new principle of informational mechanisms of the artificial intelligence. It turned out that In the process of its synthesis at macro-level of consideration (the structural and functional level) objectively is forming structure similar the known structure neocortex of the human. In addition, it turned out that at the micro-level of consideration (the level of the element base); there is absolutely no need to emulate the neurobiological element base with the help of neural networks. This allows us to solve the problem of constructing an artificial neocortex using the traditional computer element base, strictly preserving only the structural and functional similarity with the human neocortex.

In Russia there are practically no players is actively investing in artificial intelligence as a market segment. Therefore, NeocorTek Lab appeals to representatives of the international business community who are interested in the development of technologies for strong artificial intelligence, with the proposal of partnership, the options of which can be considered and discussed.

\section{CONCLUSION}

1) Currently, a significant increase in the role of geometrical methods in various fields of natural and applied sciences could be explained by the fact that mathematical analysis methods provide a quantitative characteristic of phenomena "in a small" using the infinitesimal changes their parameters. Geometrical methods, in its turn, allow obtaining the similar description of these phenomena "in general", using the large values changes of the same parameters.

2) The image forming process could be considered according to gauge theory as gauge transformation which is realized by means of the analysis of a weak video-information field representing a gauge field. Such transformation does not reflect on the observed (measurable) characteristics of physical object and ensures the adequacy of the image and of the physical component evaluation of weak video-information.

3) Gauge transformation is an over-coordinate one as does it not act on vectors and tensors of internal characteristics spaces of video-informational fields. Therefore, the geometry defined by gauge transformations is the geometry is not of spacetime but of a more general kind of space - of a fibration space.

4) There are some analogies with the psychology of human's visual processes that could be used in artificial vision. In particular, the work result of artificial vision system could be considered in the form of topical visual field in the internal (virtual, "mental") space-time of this system. This internal space and physical space-times are in the second-order isomorphism relation. At this "mental" space the representation of video-information evaluation is a reconstructed evaluation of video-information of general view, adequately collated to space-time content of objects observed in the corresponding spatial scene of the surrounding real world

5) At the artificial vision process form the hierarchy of video-information assessments as an upward hierarchy of 1D, 2D, 3D and 4D abstract images (representations) of the real world, which providing in the aggregate the harmoniousness of visual process, which physical and structural properties are considered together from the first stage of this hierarchical process of "mental" reconstruction of video-information.

6) The main function of the hierarchy of videothesauruses, at an highest level of the hierarchy of which the video-intelligence is located, is phased "strengthening" of the evaluations of video-information from a weak (rough) topology to a strong (thin) topology, which is carried out by the ascending hierarchy of AVIS.

7) At the "mental" (psychological) AVIS hierarchy level, video-intelligence is a system-forming macro-object which is considering as an ultra-high level of AVIS informational hierarchy. In set-theoretical form, video-intelligence could be considered as an aggregation of pairwise interacting 4D videoinformation evaluations or taking into account the law of idempotency as union these evaluations of video-information. 
When using the common template of informational approach, video-intelligence is presented as video-informational 4D macro-object (a measuring scale for hierarchy's ultra-high level), which used to support the interactions-measurements in "mental" 4D space of AVIS. The process is building of corresponding abstract images forming by video-intelligence could be interpreted as image thinking and it result will be an understanding of video information about the spatial content of the surrounding real world

8) Video-thesauruses (1D, 2D, 3D) in the forms of corresponding "dimensional" scales of AVIS up-ward hierarchy are formed with vision system by sequential accumulation of 1D, 2D, 3D video- experience.

9) Video-thesaurus development scheme is formed as a sequence of hypergraphs corresponded to video-thesaurus sequential development as a qualitative measuring scale. These heterarchical organizational of the video-thesaurus structures, should be formed during AVIS training process and her are rather difficult to implement. However, taking into account the law of idempotency such union of combinatorically paired organized "dimensional" video-data could degenerate into much simplier empirical qualitative measuring scale of videostructures. This scale takes the form of a fairly simple realizable generalized information hypergraph of video-thesaurus. Video-thesaurus has the structure of combinatorically organized binary system of relations between video-thesaurus elements. The theory of physical structures currently proves absent informative nontrivial theories of ternary, tetradic and other systems of relations (structures). There are only binary and unary systems of relations occurring in the nature. Thus, the considered way of development of video-intelligence by accumulating a video- experiment is the only possible.

10) The formalism of generalized information hypergraphs allows us to construct generalized hyper-graphs of video intelligence and strong intelligence, which in turn can be represented by a composition of generalized information hypergraphs of strong artificial intellects of sensory and non-sensory natures.

11)As an example, on Fig. 5 shows the structure of a simplified hypergraph of strong artificial intelligence. Here one of the partial sensory intellects, namely, video-intellect, is shown with the unfolded internal structure of the upward hierarchy of hypergraphs of the video-informations evaluations of small spatial dimensions. This hypergraph demonstrates the possibilities of the process of developing strong artificial intelligence, but not him by "depth", but by "width." Indeed, the process of developing a strong artificial intelligence is possible only on the basis of training and self-training processes, which necessarily lead to increase in the number of elements in all corresponding thesauruses and the artificial intellects (sensory and non-sensory). It is the quantitative expansion of the element composition of all these information macro-objects that is the only way of qualitative development of strong artificial intelligence.

\section{REFERENCES}

[1] E.M. Yarichin, "Informational Paradigm of Technical Vision, Pattern Recognition and Image Analysis", Vol.18, № 1, 2008, pp. 163 - 176.
[2] E.M. Yarichin, "Theory of Full (Video) Information: Bridging of the Gap between Quantity and Quality in Modern Paradigm of Information", International Journal of Information Science., vol. 3, no 3, 2013, pp.37- 58.

[3] E.M. Yarichin, "Informational Mechanism of Intellectual Technical Vision", IEEE International Siberian Conference on Control and Communications, IEEE Xplore, Digital Library, 2011, pp.84 - 86.

[4] E.M. Yarichin, "Video-information: Internal Structure and the Features of the Discretization", Inter-national Journal of Engineering and Innovative Technology (IJEIT), vol. 3, no 5, 2013, pp.133 - 140.

[5] L.D. Landau, E.M. Liphshitz, Theoretical physics. Vol. II. Field theory. Moscow: Nauka, 1988 (in Russian).

[6] A.A. Logunov, Gravity field theory. Moscow: Nauka, 2001 (in Russian).

[7] D.D. Ivanenko, G.A. Sardanashvili, Gravity. 2nd ed. Moscow: Editorial URSS, 2004 (in Russian.

[8] N.P. Konoplyova, V.N. Popov, Calibrating fields. 3d ed. Moscow: Editorial URSS, 2000 (in Russian).

[9] R. Penrose, The Road to Reality. A Complete Guide to the Laws of the Universe, Jonatan Cape, London, 2004.

[10] A.I. Berg, et. al., Control, information, intellect. Moscow: Mysl, 1976 (in Russian).

[11] H. Haken, Information and Self-organization. A Macroscopic Approach to Complex Systems, Second Enlarged Edition, Springer-Verlag Berlin Heidelberg New York, 1999,

[12] F.P. Tarasenko, Introduction to the course of information theory. Tomsk: Izdatelstvo Tomskogo Universiteta, 1963 (in Russian).

[13] B.N. Petrov, I.I. Goldenblat, G.M. Ulanov, S.V. Ulyanov, Problems of relativistic and quantum dy-namical systems control (physical and informational aspects). Moscow: Nauka, 1982 (in Russian).

[14] I.M. Kogan, Applied information theory. Moscow: Radio i svyaz, 1981 (in Russian).

[15] I.Ya. Kremer, A.I.Kremer, V.M. Petrov, Space-time processing of signals. Moscow: Radio I svyaz, 1984 (in Russian).

[16] O.N. Litvinenko, Fundamentals of radio optics. Kiev: «Tekhnika», 1974 (in Russian).

[17] V.A. Zverev, Radio optics (Transformation of signals in radio- and optics). Moscow: «Sov.radio», 1975 (in Russian).

[18] W. Thurston, D. Wicks, "Mathematics of three-dimensional manifolds", «In the world of science», Scientific American. Russ. ed., Moscow: Mir, 1984, no.9, pp.74-88 (in Russian).

[19] P. Scott, The Geometries of 3-Manifolds, London Mathematical Society, 1983.

[20] W.P. Thurston, Three-Dimensional Geometry and Topology, Edited by Levy S., Princeton, New Jersey, 1997.

[21] D. Marr, VISION. A Computational Investigation into the Human Representation and Processing of Visual Information, W.H. Freeman and Company, New York, 1982.

[22] B.K.P. Horn, Robot Vision, the MIT Press Cambridge, Massachusetts London, England, 1986.

[23] J.M. Lloyd, Thermal Imaging Systems, Plenum Press New York and London, 1975.

[24] G.S. Safronov, A.P. Safronova, Introduction to radio holography. Moscow: «Sov. radio», 1973 (in Russian).

[25] L.M. Soroko, Holography and coherent optics basics, Moscow: Nauka, 1971 (in Russian).

[26] P. Greguss, Ultrasonic imaging. Seeng by Sound, Focal Press Limited, London, Focal Press Inc., New York, 1980.

[27] Yu.G. Borisovich, N.M. Bliznyakov, Ya.A. Izrailevich, T.N. Fomenko, Introduction to topology. Moscow: Vyssh. shkola, 1980 (in Russian).

[28] E.Yu. Kondrashina, L.V. Litvintseva, D.A. Pospelov, Knowledge of time and space in intelligent sys-tems. Moscow: Nauka, 1989 (in Russian).

[29] J. Pfanzagl, in cooperation V. Baunann, H. Huber, Theory of Measurement, 2nd revised edition, Physica-Verlag, Wurzburg - Wien, 1971.

[30] J. Celko's, Joe Celko's SQL Programming Style, Elsevier Inc., 2005. 
[31] V.V. Druzhynin, D.S. Kontorov, Systematology problems (problems of complex systems theory). Moscow: «Sov. radio», 1976 (in Russian).

[32] M.A. Kholodnaya, Psychology of intellect. Paradoxes of research. 2nd ed. Saint Petersburg: Piter, 2002 (in Russian).

[33] R. Klatzy, Human Memory, W. H. Freeman and Company, San Francisco, 1975.

[34] R. Utiyama, What did physics come to (From the theory of relativity to calibrating fields theory). Russ. ed. Moscow: Znanie, 1986 (in Russian).

[35] S. - K. Chang, Principles of Pictorial Information Systems Design, Prentice-Hall International, Inc., 1989.

[36] Yu.S. Vladimirov, Metaphysics. Moscow: BINOM. Laboratory of knowledge, 2002 (in Russian).

[37] Yu.S. Vladimirov, Geometrophysics. Moscow: BINOM. Laboratory of knowledge, 2005 (in Russian).

[38] H.R. Schiffman Sensation and Perception, An Integrated Approach, Fifth Edition, John Wiley \& Sons, Inc., 2001.

[39] B.A. Dubrovin, S.P. Novikov, A.T. Fomenko, Current geometry: methods and applications. 2nd ed. Moscow: Nauka, 1986 (in Russian).
[40] A.A. Logunov, M.A. Mestvirishvili, The relativistic theory of gravity. Moscow: Nauka, 1989 (in Russian).

[41] A.A. Logunov, Gravitational field theory, Moscow: Nauka, 2001 (in Russian).

[42] Yu.I. Kulakov, Theory of physical structures. Moscow, 2004 (in Russian).

[43] V.P. Sigorskiy, Mathematical tool of an engineer. Kiev: "Tekhnika", 1975 (in Russian).

[44] Sh.-T. Yau, S. Nadis, The Shape of Inner Space, String Theory and the Geometry of the Universe's Hidden Dimensions, A Member of the Perseus Books Group, New York, 2010.

[45] J. Hawkins, S. Blakeslee, On intelligence, Times Books Henry Ford and Company, New York, 2004.

[46] R. L. Gregory, The Intelligent Eve, Wiedenfeld and Nicolson, London, 1970.

[47] R. Penrose, The Emperor's New Mind. Concerning Computers, Mind and the Laws of Physics, Oxford University Press, 1989.

[48] R. Penrose, Shadows of the Mind. A Search for the Missing Science of Consciousness,
University

Press, 